\title{
Disappearance of the de Almeida-Thouless line in six dimensions
}

\author{
M. A. Moore and A. J. Bray \\ School of Physics and Astronomy, University of Manchester, Manchester M13 9PL, UK
}

(Dated: July 24, 2021)

\begin{abstract}
We show that the Almeida-Thouless line in Ising spin glasses vanishes when their dimension $d \rightarrow 6^{+}$as $h_{A T}^{2} / T_{c}^{2}=C(d-6)^{4}\left(1-T / T_{c}\right)^{d / 2-1}$, where $C$ is a constant of order unity. It is shown that replica symmetry breaking also stops as $d \rightarrow 6^{+}$. Equivalent results that could be checked by simulations are given for the one-dimensional Ising spin glass with long-range interactions.
\end{abstract}

PACS numbers: 75.50.Lk, 75.40.Cx, 05.50.+q

\section{INTRODUCTION}

The field of spin glasses is now well into its fourth decade, with many important questions still unresolved. Chief among these is the nature of the ordered phase. The principal rival theories of it are (i) the replicasymmetry-breaking (RSB) theory of Paris ${ }^{1}$, motivated by the exact solution of the Sherrington-Kirkpatrick (SK) mean-field model, and (ii) the droplet/scaling theory 2 2/3 based on the properties of excitations in the ordered phase. There is still no general consensus on which (if any) of these theories is correct.

An important (and perhaps the simplest) discriminator between the theories is the predicted behavior of the system when the temperature is decreased in the presence of an applied magnetic field. According to the RSB scenario, there will still be a phase transition in an applied field $h$, ocurring at a temperature $T_{c}(h)$, the Almeida-Thouless (AT) line ${ }^{4}$, which decreases as the field increases. This line can be explicitly calculated in the mean-field SK model. For small $h$ it has the form $T_{c}(h)=T_{c}(0)-$ const. $h^{2 / 3}$ or, equivalently,

$$
h_{A T}^{2} \propto\left[T_{c}-T\right]^{3},
$$

$\left(T_{c}(0) \equiv T_{c}\right)$. In the RSB theory, this line is the phase boundary between the (high-temperature) replica symmetric phase and the (low-temperature) broken replica symmetry spin glass phase. Within the droplet/scaling theory, on the other hand, there is no such line: An applied magnetic field is predicted to remove the phase transition completely and the low-temperature phase in the absence of a field has replica symmetry.

Before presenting our calculation, we recall that the shape of the AT line starts to differ from Eq. (1) already for $d<8$, as shown by Green et al ${ }^{[5}$ and by Fisher and Sompolinsky 6 . Instead of Eq. 11, these authors show that the AT line has the form

$$
h_{A T}^{2} \propto\left[T_{c}-T\right]^{d / 2-1}
$$

for $6<d<8$. Note that as $d \rightarrow 8^{-}$in Eq. (2), Eq. (1) is recovered.

In this paper we will derive an exact result for the form of the AT line in $d=6+\epsilon$ dimensions, correct to leading non-trivial order in $\epsilon$. The result can be written, for $T$ close to $T_{c}(0)$, as

$$
\frac{h_{A T}^{2}}{T_{c}^{2}}=C(d-6)^{4}\left(1-\frac{T}{T_{c}}\right)^{d / 2-1},
$$

where $C$ is a non-universal constant. The form is the same as that proposed by Green at al. and by Fisher and Sompolinsky, but the amplitude contains the factor $(d-6)^{4}$ which vanishes (rapidly) for $d \rightarrow 6$.

In itself this result does not prove that there is no AT line below six dimensions. If there were an AT line below six dimensions, it would have the scaling form $h_{A T}^{2} \sim\left(1-T / T_{c}\right)^{\beta+\gamma}$, where $\beta$ and $\gamma$ are the critical exponents of the zero-field spin glass 6 . The vanishing of the amplitude in Eq. (3) would arise to ensure continuity in six dimensions of the forms above and below $T_{c}^{77}$. Hence we need to provide an additional argument why there should be no AT line when $d \leq 6$.

Even before the droplet/scaling theory had been developed, Bray and Roberts ${ }^{8}$ (BR) had used standard renormalization group (RG) methods to investigate the putative RG fixed point that controls the critical behavior at the AT line. In zero field there are three degenerate soft modes at the critical point (usually called the "longitudinal", "anomalous" and "replicon" modes). A conventional RG analysis9 shows that the upper critical dimension is $d_{u}=6$. For $d>6$ the Gaussian fixed point is stable and the critical exponents take their mean-field values. For $d<6$, a non-trivial fixed point is stable and non-classical exponents are obtained and can be calculated as a power series in $(6-d)$ in the conventional way.

An applied magnetic field, however, changes everything. The field lifts the degeneracy, leaving a single soft mode, the replicon mode. In their RG calculation, BR discarded the two hard modes, and derived RG recursion relations appropriate to the soft modes which we discuss below. Again, the critical dimension is $d_{u}=6$. There are now two coupling constants $w_{1}$ and $w_{2}$. In dimensions $d>6$, the RG flows have a single stable fixed point, the Gaussian fixed point with $w_{1}^{*}=w_{2}^{*}=0$, implying that for $d>6$ there is a continuous phase transition, with critical exponents given by their mean-field values.

For $d \leq 6$, however, no physical stable points could be found. BR suggested that this might be because there was no AT line for $d \leq 6$. We shall strengthen this argument by examining the RG flows when $d>6$ in more 
detail. We find that the basin of attraction of the Gaussian fixed point shrinks to zero as $\epsilon \rightarrow 0$; it has a linear extent of order $\epsilon^{1 / 2}$. It thus seems natural to expect that there is no AT line when $d \leq 6$ : There is no physical stable fixed point when $d \leq 6$ and the basin of attraction for the controlling fixed point in $6+\epsilon$ dimensions is shrinking to zero as $\epsilon \rightarrow 0$.

Above the AT line the high-temperature phase is replica symmetric. Below it, the phase has broken replica symmetry. Thus if the AT line is disappearing as $d \rightarrow 6^{+}$, then one would naturally expect that replica symmetry breaking in the zero-field case would vanish as $d \rightarrow 6^{+}$. We shall demonstrate that is indeed the case by showing that the "breakpoint" $x_{1}$ in Parisi's RSB function $q(x)^{1}$ goes to zero in this limit. Thus the low-temperature phase for $d \leq 6$ should be replica symmetric.

The structure of the paper is as follows. In section II, we present an RG analysis, valid for $d \geq 6$, that leads directly to Eq. (3). In section III we analyse the consequences of our RG results for the form of the breakpoint, $x_{1}$, in the Parisi RSB theory. We find the $x_{1}$ tends to zero for $d \rightarrow 6^{+}$, suggesting that replica symmetry breaking goes away in six dimensions. In section IV, the RG equations of BR are presented. The basin of attraction of the Gaussian fixed point is determined numerically. It is a compact region enclosing the origin, with linear dimensions of order $\sqrt{\epsilon}$, shrinking to a point as $d \rightarrow 6^{+}$. In section $\mathrm{V}$, we treat the one-dimensional spin-glass with long-range inetractions falling off with distance $r$ as $r^{-\sigma}$. This model has mean-field behavior for $\sigma<2 / 3$ and nonclassical behavior for $\sigma>2 / 3$. Thus $\sigma<2 / 3$ corresponds to $d>6$ in the short-ranged model. We show that the de Almeida-Thouless line goes away as $\sigma \rightarrow 2 / 3^{-}$, and that the breakpoint $x_{1}$ of the Parisi function goes to zero in this limit. We conclude with a brief discussion of the main points in section VI.

\section{RENORMALIZATION GROUP ANALYSIS}

We now describe the calculations that lead to the result quoted above.. We start from the Ginzburg-LandauWilson free-energy functional for the Ising spin glass which, written in terms of the replica order parameter field, is

$$
\begin{aligned}
& F\left[\left\{Q_{\alpha \beta}\right\}\right]=\int d^{d} x\left[\frac{1}{2} r \sum_{\alpha<\beta} Q_{\alpha \beta}^{2}+\frac{1}{2} \sum_{\alpha<\beta}\left(\nabla Q_{\alpha \beta}\right)^{2}\right. \\
& \left.+\frac{w}{6} \sum_{\alpha<\beta<\gamma} Q_{\alpha \beta} Q_{\beta \gamma} Q_{\gamma \alpha}-h^{2} \sum_{\alpha<\beta} Q_{\alpha \beta}+O\left(Q^{4}\right)\right](4)
\end{aligned}
$$

where $h$ is the applied field. We use conventional RG methods 9 , but work above the upper critical dimension, $d_{u}=6$, so we define $\epsilon=d-6$. A simple scaling analysis of the terms in the functional of Eq. (4) shows that the natural size of $h^{2}$ is $\sim|r|^{2} / w$; this remains the correct scaling form for all $d>6 . h_{A T}^{2}$ is given by Eq. (3) and Eq. 16 below, and is much smaller than $|r|^{2} / w$ provided $w^{2}|r|^{\epsilon / 2} \ll 1$. We shall work in this limit as it allows the use of the simple RG equations of the $h=0$ theory to obtain the AT line as $T \rightarrow T_{c}$. When $d \leq 6$ this will not be possible and the full set of RG equations in Ref 10 would have to be solved instead.

The renormalization group flows for $w$ and $r$ read ${ }^{10}$,

$$
\begin{aligned}
\frac{d w}{d l} & =\frac{1}{2}[-\epsilon-3 \eta(l)] w-2 w^{3} \\
\frac{d r}{d l} & =[2-\eta(l)] r-4 w^{2} r,
\end{aligned}
$$

while $h^{2}$ evolves according to

$$
\frac{d\left(h^{2}\right)}{d l}=\frac{d+2-\eta(l)}{2} h^{2}
$$

where $\eta(l)=-(2 / 3) w(l)^{2}$. The RG equations are correct to $O\left(w^{3}\right)$, and to linear order in $r$. There is an additional $r$-independent term of order $w^{2}$ in Eq. (6) that we have omitted since it ultimately just leads to a shift in $r$, i.e. a shift in the critical temperature. In these equations the usual geometrical factor $K_{d}=2 / \Gamma(d / 2)(4 \pi)^{d / 2}$ has been absorbed into $w^{2}$. For $d>6$ the Gaussian fixed point is stable and the critical exponents take their mean-field values.

Integrating the RG flow equations when $d>6$ up to scale $l$ gives

$$
\begin{aligned}
w(l) & =\frac{w(0) \exp (-\epsilon l / 2)}{\left[\left(2 w(0)^{2} / \epsilon\right)\left(1-e^{-\epsilon l}\right)+1\right]^{1 / 2}} \\
r(l) & =r(0) \exp [2 l-(10 / 3) \Delta(l)] \\
h(l)^{2} & =h(0)^{2} \exp [\{(d+2) / 2\} l+(1 / 3) \Delta(l)]
\end{aligned}
$$

where

$$
\Delta(l)=\int_{0}^{l} w\left(l^{\prime}\right)^{2} \mathrm{~d} l^{\prime}=\frac{1}{2} \ln \left[\frac{2 w(0)^{2}}{\epsilon}\left(1-e^{-\epsilon l}\right)+1\right]
$$

and $w(0)=w, h^{2}(0)=h^{2}, r(0)=r$.

At large $l$,

$$
w(l) \rightarrow\left[\frac{\epsilon}{2\left(1+\epsilon / 2 w(0)^{2}\right)}\right]^{1 / 2} \exp (-\epsilon l / 2) .
$$

The exponential follows from the $O(w)$ terms in the RG equation (5). The $O\left(w^{3}\right)$ terms in Eq. (5) serve to fix the amplitude of the asymptotic decay. The basic idea is to integrate the RG equations to a scale $l^{*}$ at which the running coupling constant, $w\left(l^{*}\right)$, is small enough for one-loop order perturbation theory to be accurate.

The one-loop perturbative calculation of the AT line has been carried out by Green et al.. The result is

$$
h^{2} / Q=144 w^{2}|r|^{2} I_{d}
$$

where $I_{d}$ is the integral $\int_{0}^{\infty} d^{d} q q^{-4}\left(q^{2}+|r|\right)^{-2}$, which equals $A_{d}|r|^{d / 2-4}$ and $A_{d}=1 / 2$ for $d=6$. In Eq. 13 , the geometrical factor $K_{d}$ has again been absorbed into $w^{2}$, and $Q$ is the mean-field value of the order parameter 
for $r<0$. The factor of 36 between this result and that in ${ }^{5}$ is due to the different definitions of $w$, which differ by a factor of 6 in the two calculations. Using the result $Q \sim$ $3|r| / w$, which holds on the AT line in the perturbative limit where $w^{2}|r|^{\epsilon / 2} \ll 1$, we find

$$
h^{2} \sim w|r|^{d / 2-1}, \quad(6<d<8),
$$

which is the result obtained in Refs $\frac{5}{5}$ and $\frac{\sqrt{6}}{}$.

To exploit the perturbation theory result, we coarsegrain to scale $l$ and replace $w$ by $w(l), r$ by $r(l)$ and $h^{2}$ by $h(l)^{2}$ to obtain $h(l)^{2} \sim w(l)|r(l)|^{d / 2-1}$. Eq. $12 p$ shows that $w(l)$ becomes progressively smaller as $l$ increases. Our use of the perturbative result will become valid at some value $l=l^{*}$, where $l^{*}$ will be specified below. Inserting the $l$ dependent forms for $h(l), w(l)$ and $r(l)$ at $l=l^{*}$, one obtains

$$
h(0)^{2} \equiv h_{A T}^{2}=\frac{w(0)|r(0)|^{d / 2-1}}{\left[\left(2 w(0)^{2} / \epsilon\right)\left(1-e^{-\epsilon l^{*}}\right)+1\right]^{5 d / 6-1}} .
$$

Setting $d=6+\epsilon$, and taking $\exp \left(-\epsilon l^{*}\right) \ll 1$ we obtain the final result, correct to leading order in $\epsilon$, that

$$
h_{A T}^{2} \sim\left(\frac{\epsilon}{2 w(0)^{2}}\right)^{4} w(0)|r(0)|^{d / 2-1} \equiv\left(\frac{\epsilon}{2 w^{2}}\right)^{4} w|r|^{d / 2-1} .
$$

This is equivalent to Eq. (3).

One cannot simply set $l^{*} \rightarrow \infty$ in Eq. (15). The RG equations as presented here are valid provided $r\left(l^{*}\right)$ remains small or comparable to the square of the cut-off in the theory, which is conventionally taken to be unity. To leading order $r\left(l^{*}\right)=r(0) \exp \left(2 l^{*}\right)$, so we shall fix $l^{*}$ by setting 6

$$
\left|r\left(l^{*}\right)\right| \approx|r(0)| e^{2 l^{*}}=1 .
$$

Eq. 16 will hold provided that

$$
e^{-\epsilon l^{*}}=|r(0)|^{\epsilon / 2} \equiv|r|^{\epsilon / 2} \ll 1 .
$$

Thus as $\epsilon \rightarrow 0$, the temperature interval near $T_{c}$ over which Eq. (3) is accurate becomes very narrow.

\section{DOES RSB DISAPPEAR FOR $d<6$ ?}

We are arguing in this paper that the lower critical dimension for the AT line - the line beneath which RSB sets in in the presence of a field - is 6 . In zero field there is still a phase transition when $d \leq 6$, provided $d>d_{l}$, where $d_{l}$ is the lower critical dimension, thought to be between 2 and 3 . One might wonder whether replica symmetry breaking is present in the zero-field phase or whether RSB too disappears below six dimensions. We next present an argument that RSB in zero field vanishes as $d \rightarrow 6^{+}$based on the same methods which we used to derive the dimensionality dependence of the AT line.

When $d>6$ the form of replica symmetry breaking is well-established ${ }^{1111}$. $\left\langle Q_{\alpha \beta}\right\rangle$ becomes a function $q(x)$ in the interval $1 \geq x \geq 0$, which is constant for $1 \geq x \geq x_{1}$ at the value $q_{E A}(=Q)$ and then falls from this value to zero at $x=0$. For the functional of Eq. (4) (with $h^{2}$ set to zero) the breakpoint $x_{1}$ is given to one-loop order for $6<d<8$ by 11

$$
x_{1} \sim w^{2}|r|^{d / 2-3} .
$$

If $x_{1}$ were zero, the spin glass would be replica symmetric. We shall argue that as $d \rightarrow 6^{+}, x_{1}$ goes to zero linearly with $(d-6)$, suggesting that when $d \leq 6$ there will be no replica symmetry breaking. Higher loop terms will leave the exponent of $|r|$ unchanged, but modify its prefactor, just as they do for the AT line of Eq. (2).

We shall work in $6+\epsilon$ dimensions again with $\epsilon$ small and take $|r|$ small so as to permit neglect of the quartic terms in the functional Eq. (4). The RG will be used to coarse-grain to scale $l^{*}$ at which the form of the perturbative one-loop order expression for the breakpoint $x_{1}$ becomes valid. The breakpoint $x_{1}[r, w]$ has zero scaling dimension, so it follows that

$$
x_{1}[r, w]=x_{1}\left[r\left(l^{*}\right), w\left(l^{*}\right)\right] \sim w\left(l^{*}\right)^{2}\left|r\left(l^{*}\right)\right|^{d / 2-3} .
$$

Inserting our previous expressions for $r\left(l^{*}\right)$ and $w\left(l^{*}\right)$, one finds

$$
x_{1}[r, w] \sim \frac{w(0)^{2}|r(0)|^{d / 2-3}}{\left[\left(2 w(0)^{2} / \epsilon\right)\left(1-e^{-\epsilon l^{*}}\right)+1\right]^{5 d / 6-4}} .
$$

$l^{*}$ is specified as in Eq. (18). Hence in the limit when $\epsilon$ is small (but $\epsilon l^{*} \gg 1$ )

$$
x_{1} \sim \frac{1}{2}(d-6)|r|^{d / 2-3},
$$

which goes to zero as $d \rightarrow 6^{+}$, implying that replica symmetry breaking vanishes in this limit.

\section{THE BRAY-ROBERTS CALCULATION AND THE ISLAND OF STABILITY}

We shall now describe the BR calculation of the RG equations pertinent to the AT line. It is this work which is at the heart of our contention that 6 is the lower critical dimension for the existence of the AT line. As mentioned before, their approach is to study just the fields in the replicon sector $\tilde{Q}_{\alpha \beta}$, which are such that $\sum_{\beta} \tilde{Q}_{\alpha \beta}=0$. The effective functional is

$$
\begin{gathered}
F\left[\left\{\tilde{Q}_{\alpha \beta}\right\}\right]=\int d^{d} x\left[\frac{1}{4} \tilde{r} \sum \tilde{Q}_{\alpha \beta}^{2}+\frac{1}{4} \sum\left(\nabla \tilde{Q}_{\alpha \beta}\right)^{2}\right. \\
\left.+\left(w_{1} / 6\right) \sum \tilde{Q}_{\alpha \beta} \tilde{Q}_{\beta \gamma} \tilde{Q}_{\gamma \alpha}+\left(w_{2} / 6\right) \sum \tilde{Q}_{\alpha \beta}^{3}\right] .
\end{gathered}
$$

Here the convention has been adopted that the sums over replica indices are unrestricted except that $\tilde{Q}_{\alpha \alpha}=0$. At the AT line, $\tilde{r}=0$ in the mean-field approximation. According to BR, the coupling constants $w_{1}$ and $w_{2}$ would have for $d>6$ the RG flow equations

$$
\frac{d w_{1}}{d l}=\frac{1}{2}\left[-\epsilon-3 \eta_{R}(l)\right] w_{1}+14 w_{1}^{3}-36 w_{1}^{2} w_{2}+18 w_{1} w_{2}^{2}+w_{2}^{3}
$$




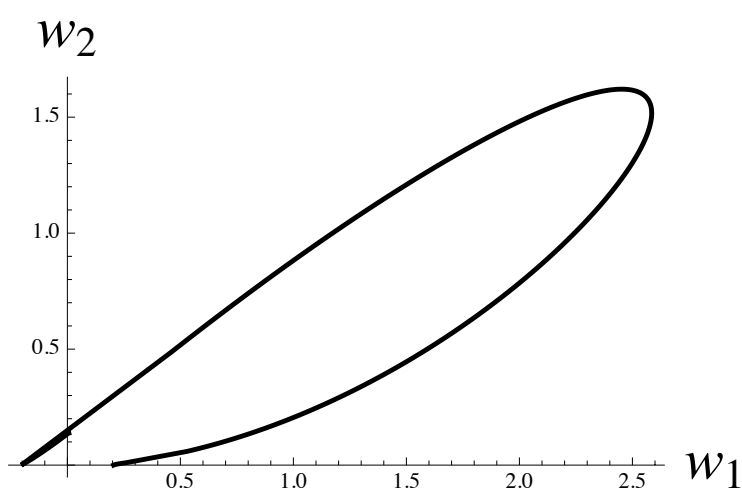

FIG. 1: The island of stability of the Gaussian fixed point for $d>6$. Distances are measured in units of $\sqrt{\epsilon}$. Only the region $w_{2}>0$ is displayed.

$$
\frac{d w_{2}}{d l}=\frac{1}{2}\left[-\epsilon-3 \eta_{R}(l)\right] w_{2}+24 w_{1}^{2} w_{2}-60 w_{1} w_{2}^{2}+34 w_{2}^{3} .
$$

Once again we have adopted the convention of absorbing the geometric factor $K_{d}$ into $w_{1}$ and $w_{2}$ and $\eta_{R}(l)=$ $\left(4 w_{1}^{2}-16 w_{1} w_{2}+11 w_{2}^{2}\right) / 3$. Presumably one could obtain these replicon sector equations by integrating the full equations of Ref ${ }^{[10}$ containing the hard longitudinal and anomalous modes as well as the replicon modes until the hard modes are decoupled from those in the replicon sector. If one were able to carry out this formidable task the initial values of $w_{1}$ and $w_{2}$ could be determined. We suspect that the initial value of $w_{1}$ would turn out to be of order $w\left(l^{*}\right)\left(\sim \epsilon^{1 / 2}|r|^{\epsilon / 4}\right)$. At mean-field level $w_{2}$ is $\sim y Q$, where $y$ is the coefficient of a particular quartic term, $(y / 4) \sum_{\alpha<\beta} Q_{\alpha \beta}^{4}$, found when one goes beyond the cubic functional in Eq. 44 8 . The effective value of $y$ is dominated at small $|r|$ when $6<d<8$ by its renormalization by the four cubic vertex "box" diagram ${ }^{6}$. Then $y \sim$ $w^{4}|r|^{d / 2-4}$ and so $w_{2} \sim w\left(l^{*}\right)^{3}\left|r\left(l^{*}\right)\right|^{\epsilon / 2}\left(\sim \epsilon^{3 / 2}|r|^{3 \epsilon / 4}\right)$. Fortunately we do not need the precise initial values of $w_{1}$ and $w_{2}$ for our argument, which is based upon the form of the basin of attraction of the Gaussian fixed point of Eqs. (24)- 25).

The basin of attraction has been determined numerically, and is displayed in Figure 1. By scaling both $w_{1}$ and $w_{2}$ by $\epsilon^{1 / 2}$ and writing $\tilde{l}=\epsilon l$, the explicit dependence on $\epsilon$ in Eqs. 24-25) can be removed. There is a peculiarly shaped compact region (the "island of stability") around the origin in the $w_{1}, w_{2}$ space inside which all flows are to the Gaussian fixed point $w_{1}^{*}=0=w_{2}^{*}$. Outside this region the flows are to infinity. The linear extent of this basin of attraction is from the scaling of $w_{1}$ and $w_{2}$ of order $\epsilon^{1 / 2}$. Thus as $\epsilon \rightarrow 0$, the size of the basin of attraction shrinks to zero. Now BR showed that when $d \leq 6$ there was no stable physical fixed point; all flows of $w_{1}$ and $w_{2}$ were to infinity. Since the basin of attraction of the fixed point associated with the AT line is shrinking to zero as $d \rightarrow 6^{+}$and no physical stable fixed points exists for $d \leq 6$, it seems natural to expect that the lower critical dimension of the AT line and replica symmetry breaking generally must be 6 . This is a result in line with earlier expectations ${ }^{3}$ but is also in accord with more recent results for the "stronglydisordered spin-glass model", a model with an unusual bond-distribution 12 .

The behavior of the basin of attraction of the Gaussian fixed point of Eqs. (24)- 25$)$ as $d \rightarrow 6^{+}$should be contrasted with that of Eq. (5) for the zero-field case. In the latter case the basin does not shrink to zero as $d \rightarrow 6^{+}$ and there is a phase transition when $d_{l}<d \leq 6$.

Our treatment of the AT line was done using the zerofield RG equations with one coupling constant $w$ rather than the full set of coupling constants, $w_{1}, w_{2}, \cdots, w_{8}$ of Ref ${ }^{10}$. The justification for this is that all but $w_{1}$ can be dropped when obtaining the form of the AT line as $T \rightarrow T_{c}$. To elaborate this point further we have already quoted forms for $w_{2}\left(l^{*}\right)$ and $w_{1}\left(l^{*}\right)$ and as $|r| \rightarrow 0, w_{2}\left(l^{*}\right)$ is indeed neglible in comparison with $w_{1}\left(l^{*}\right)$. However for $d \leq 6$, the situation is quite different. If the coupling coefficients $w_{2}, w_{3}, \cdots, w_{8}$ are anything but zero, the RG flows will take them to infinity and so for $d \leq 6$ it is never possible to work with just the one-coupling constant RG equations. The same point is relevant for the attempts to extend RSB calculations to $d \leq 6$ in zero-field $\frac{11}{11}$. For $d>6$ all the coupling constants can flow to the Gaussian fixed point.

\section{THE ONE-DIMENSIONAL SPIN GLASS WITH LONG-RANGED INTERACTIONS}

Because our calculation on the form of the AT line is only valid when $\epsilon$ is small, there is no chance that it can be directly checked by simulations. However, we can derive the analogous results for the one-dimensional Ising spin glass with long-range interactions, whose AT line has recently been the subject of contradictory numerical simulations 13114. The Hamiltonian of these studies are variants of

$$
H=-\sum_{<i j>} J_{i j} S_{i} S_{j}-\sum_{i} h_{i} S_{i}
$$

where $h_{i}$ is a random field of variance $h^{2}$, the sum is over all pairs $\langle i j\rangle$, and $i$ and $j$ are positions on the one-dimensional lattice. The interaction

$$
J_{i j}=J \frac{\epsilon_{i j}}{|i-j|^{\sigma}},
$$

where the $\epsilon_{i j}$ are independent random variables with a Gaussian distribution of zero mean and unit variance. This model was introduced by Kotliar et al.15, who showed that for $\sigma<2 / 3$ the model has meanfield critical exponents, and non-mean field exponents for $2 / 3<\sigma<1$. When $\sigma>1$, there is no finite temperature phase transition. The $\sigma$-interval, $2 / 3<\sigma<1$, is the analogue for short-range spin glasses of the dimension range between the upper critical dimension $\left(d_{u}=6\right)$ and the 
lower critical dimension, while $\sigma<2 / 3$ corresponds to dimensionalities $d>6$. Thus just by changing the value of $\sigma$ one can explore both systems corresponding to high and low dimensionality.

Our expectation is that when $\sigma<2 / 3$ there will be an AT line and both Refs $\frac{13 \mid 14}{13}$ confirm this. Unfortunately for $\sigma>2 / 3$ the two groups of simulators were in disagreement with each other: Ref $\frac{13}{13}$ did not see an AT transition, whereas Ref ${ }^{14}$ did. Our prediction below of the form of the AT line as $\sigma \rightarrow 2 / 3^{-}$supports the conclusions of Ref. 13 .

The RG equations near the upper critical value of $\sigma$, $2 / 3$, were first written down in Ref.15. They exploited the fact that for these long-range interactions $2-\eta=$ $2 \sigma-1^{16}$. The bare propagator is $1 /\left(q^{2 \sigma-1}+r\right)$. The RG flow equations become

$$
\begin{aligned}
& \frac{d w}{d l}=-(2-3 \sigma) w-2 w^{3} \\
& \frac{d r}{d l}=(2 \sigma-1) r-4 w^{2} r
\end{aligned}
$$

while $h^{2}$ grows as

$$
\frac{d\left(h^{2}\right)}{d l}=\sigma h^{2} .
$$

Eqs. (28) and (29) are valid only when $w$ is small, but Eq. (30) is exact.

The perturbative calculation along the lines of Green et al ${ }^{5}$ of the AT line to one loop order gives a result similar to Eq. (14):

$$
h^{2} / Q=144 w^{2}|r|^{2} I_{\sigma},
$$

where $I_{\sigma}=\int_{0}^{\infty} d q q^{-2(2 \sigma-1)}\left(q^{2 \sigma-1}+|r|\right)^{-2}$, which equals $B_{\sigma}|r|^{(5-8 \sigma) /(2 \sigma-1)}$, and $B_{2 / 3}=3$. Hence to one-loop order the equation of the AT line is

$$
h^{2} \sim w|r|^{\frac{2-2 \sigma}{\sigma-1}}, \quad(2 / 3>\sigma>5 / 8) .
$$

For $\sigma \rightarrow 5 / 8$, the mean-field AT form $h^{2} \propto|r|^{3}$ is recovered. Hence $\sigma=5 / 8$ is the analogue of 8 dimensions for short-range spin glasses.

It is straightforward to integrate the RG equations for the long-range case to obtain the form of the AT line as $\sigma \rightarrow 2 / 3^{-}$. The result is

$$
h(0)^{2} \sim\left(\frac{2-3 \sigma}{w(0)^{2}}\right)^{\frac{3-2 \sigma}{2(2 \sigma-1)}} w(0)|r(0)|^{\frac{2-2 \sigma}{2 \sigma-1}}
$$

or equivalently

$$
\frac{h_{A T}^{2}}{T_{c}^{2}}=C(2-3 \sigma)^{\frac{3-2 \sigma}{2(2 \sigma-1)}}\left(1-\frac{T}{T_{c}}\right)^{\frac{2-2 \sigma}{2 \sigma-1}},
$$

and $C$ is again a constant of $O(1)$. Hence as $\sigma \rightarrow 2 / 3^{-}$ the AT line goes away. That there was no AT transition in the interval $1>\sigma>2 / 3$ has also recently been suggested $^{[17}$ from an expansion about $\sigma=1$, the "lower critical value".

The equivalent result for the "break-point", $x_{1}$, in the Parisi function for the one-dimensional long-ranged system is

$$
x_{1} \sim(2-3 \sigma)|r|^{(4-6 \sigma) /(2 \sigma-1)},
$$

when $\sigma \rightarrow 2 / 3-$.

\section{CONCLUSION}

We have presented arguments that the AlmeidaThouless line in spin glasses is absent in systems with six or fewer space dimensions, i.e. these systems exhibit no phase transition under cooling if an external magnetic field is present. We have also argued that the features associated with broken replica symmetry, in the Parisi solution of the SK model, are not present in finitedimensional spin glasses with $d \leq 6$.

Equivalent results have been obtained for a onedimensional spin glass with interactions decaying with distance $r$ as $1 / r^{\sigma}$. These systems are more amenable to simulation than short-ranged systems in highdimensional space. To date, however, there seem to be no simulational studies of the one-dimensional long-range model which might help to confirm Eq. (34) in the interval $5 / 8<\sigma<2 / 3$. One issue which will complicate such studies was pointed out in Ref ${ }^{13}$. Simulations at the AT line require the system to be large enough so that the Parisi overlap function $P(q)$ has only positive support. This requires $h(Q N)^{1 / 2}>T$. Whether simulations can be done with the number of spins $N$ large enough to meet this requirement remains to be seen.

The debate as to the nature of the spin glass phase in three dimensional systems has run for so long because on the experimental side, for example, dynamical effects can produce an apparent AT line $e^{3118}$, while in simulations there are always finite size effects which can mimic some of the effects of replica symmetry breaking $\frac{19}{19}$. As a consequence it was always hard to be certain which of the two pictures, RSB or droplet scaling, was correct. Ref.20 is an example of a recent simulation and provides further references. We believe that our calculations provide strong arguments that the spin glass phase will not have replica symmetry breaking in dimensions $d \leq 6$.

\section{ACKNOWLEDGEMENTS}

We are indebted to G. Parisi, T. Temesvari, and A. P. Young for their useful comments. 
1 G. Parisi, Phys. Rev. Lett. 43, 1754 (1979); J. Phys. A 13, 1101 (1980); ibid. 13, 1887 (1980); Phys. Rev. Lett. 50, 1946 (1983); M. Mézard, G. Parisi, N. Sourlas, G. Toulouse, and M. Virasoro, Phys. Rev. Lett. 52, 1156 (1984).

2 D. S. Fisher and D. A. Huse, Phys. Rev. Lett. 56, 1601 (1986); Phys. Rev. B 38, 386 (1988); ibid. 38, 373 (1988).

3 A. J. Bray and M. A. Moore, Lecture Notes in Physics 275, 121 (1986).

4 J. R. L. de Almeida and D. J. Thouless, J. Phys. A 11, 983 (1978).

5 J. E. Green, M. A. Moore, and A. J. Bray, J. Phys. C 16, L815 (1983).

${ }^{6}$ D. S. Fisher and H. Sompolinsky, Phys. Rev. Lett. 54, 1063 (1985).

7 A. P. Young, private communication.

8 A. J. Bray and S. A Roberts, J. Phys. C 13, 5405 (1980).

9 A. B. Harris, T. C. Lubensky, and J. H. Chen, Phys. Rev. Lett. 36, 415 (1976).

10 I. R. Pimentel, T. Temesvári, and C. De Dominicis, Phys.
Rev. B 65224420 (2002).

11 T. Temesvari, C. De Dominicis, and I. Kondor, Eur. Phys. J. B 11, 629 (1999).

12 T. S. Jackson and N. Read, Phys. Rev. E 81, 021130 (2010).

13 H. G. Katzgraber, D. Larson, and A. P. Young, Phys. Rev. Lett. 102, 177205 (2009).

14 L. Leuzzi, G. Parisi, R. Ricci-Tersenghi, and J. J. RuizLorenzo, Phys. Rev. Lett. 103, 267201 (2009).

15 G. Kotliar, P. W. Anderson, and D. L. Stein, Phys. Rev. B 27, R602 (1983).

16 J. Sak, Phys. Rev. B 8, 281 (1973).

17 M. A. Moore, Phys. Rev. B 82, 014417 (2010).

18 J. Mattsson, T. Jonsson, P. Nordblad, H. A. Katori, and A. Ito, Phys. Rev. Lett. 74, 4305 (1995).

19 A. J. Bray and M. A. Moore, J. Phys. A18, L683 (1985).

20 T. Jörg, H. G. Katzgraber, and F. Krzakala, Phys. Rev. Lett. 100, 197202 (2008). 